\title{
Gas inflow and nuclear star formation in galaxies with non-axisymmetric bulges
}

\author{
E. Kim ${ }^{1}$, S. S. Kim ${ }^{1,2}$, G.-H. Lee ${ }^{3}$, \\ M. G. Lee ${ }^{3}$ and R. de Grijs ${ }^{4}$ \\ ${ }^{1}$ School of Space Research, Kyung Hee University, Yongin, Gyeonggi 446-701, Korea \\ email: ebkim@khu.ac.kr \\ ${ }^{2}$ Department of Astronomy \& Space Science, Kyung Hee University, \\ Gyeonggi 446-701, Korea \\ ${ }^{3}$ Department of Physics and Astronomy, Seoul National University, \\ Gwanak-gu, Seoul 151-742, Korea \\ ${ }^{4}$ Kavli Institute for Astronomy \& Astrophysics and Department of Astronomy, \\ Peking University, Yi He Yuan Lu 5, Hai Dian District, Beijing 100871, China
}

\begin{abstract}
We present the dependence of the amount of nuclear star formation on the nonaxisymmetry of a bulge of disk galaxies. For this, we use a volume-limited sample of spiral galaxies at $0.02<=\mathrm{z}<0.055$ from the SDSS DR7. Among 3173 final sample galaxies with an axis ratio $\mathrm{b} / \mathrm{a}>0.6$ and a bulge fraction ranged in $\mathrm{B} / \mathrm{T}<=0.41$, nuclear starburst galaxies are $10 \%$. We find that a fraction of the nuclear starburst galaxies become higher when ellipticity of a bulge increases in early type galaxies. Also, the fraction increases clearly when early type galaxies are isolated and in low density region. Our results indicate that the non-axisymmetry of bulges assists gas to fall inside and affects the nuclear starburst process in disk galaxies.
\end{abstract}

Keywords. Galaxies:sprial — Galaxy:bulge — Galaxies: starburst

\section{Introduction}

Gas inflow in a galaxy is a key process during galactic evolution. The migration of gases takes place by tidal interactions and mergers or non-axisymmetric mass distribution in the galactic nucleus gravitational potential. A large amount of inward gas migration can induce nuclear star formation. In the second case, it is well known that non-axisymmetric bar torque drives gas inflows; however, research of a relation between non-axisymmetry of the bulge and nuclear star formation has not been well studied observationally. In this study, we present that ellipticity of bulges showing non-axisymmetry and nuclear starbursts have a correlation with using SDSS DR7. This relation is dependent on the Hubble type and environmental factors.

\section{Sample selection and data analysis}

Firstly, we used volume limited r-band late-type galaxies from SDSS DR7 ranged in $\mathrm{Mr}<-19.5,0.02<=\mathrm{z}<=0.05489$ and axis ratio $\mathrm{b} / \mathrm{a}>0.6$ for elongated bulges on the view of face-on disks, and only selected galaxies with no warning flag in their spectrum (zwaring $=0$, sciencePrimary $=1$ ). Then we separated the sample to the nuclear nonstarburst and starburst galaxies located in the star-forming region of the BPT diagram with an equivalent width of $\mathrm{H} \alpha$ greater than $50 \AA$ (Abazajian et al. 2009, Choi et al. 2010). Secondly, we matched the sample to the catalog of the bulge/disk decompositions 

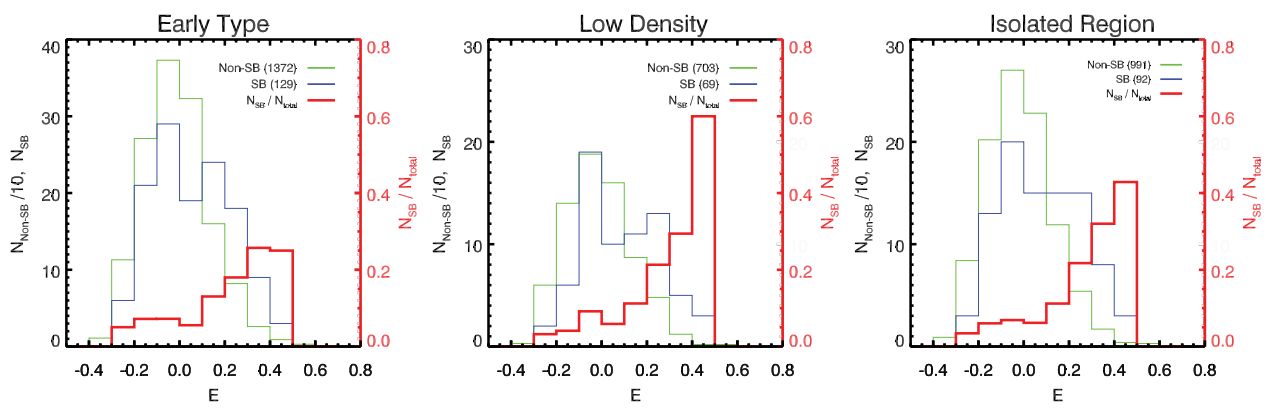

Figure 1. Distribution of nuclear star forming and non-star forming galaxies on the $\mathrm{E}$ in galaxies with $0.15<\mathrm{B} / \mathrm{T}<=0.41$. Green lines show the number of non-starburst galaxies divided by 10 , the red lines indicate the number of nuclear starburst galaxies, and the blue lines show the fraction of Non-SB/ SB galaxies of each ellipticity bin on right vertical axis. Left to right sample is early type disk galaxies, those in a low density and isolated region. [A COLOR VERSion is AVAILABle ONLINE.]

from the Legacy area of SDSS DR7 (Simard et al. 2011) providing a bulge semi-major axis effective radius of bulge and bulge fraction (B/T), etc. The sum of an exponential disk and a de Vaucouleurs bulge (Sersic index $n=4$ ) was used as a galaxy image model, and we excluded the sample with a bad fitting probability $\left(\mathrm{P}_{p S}>0.32\right)$ and an effective radius lower than 1 pixel. Third, environmental factors of galaxies from Park \& Choi (2008), which are mass density of twenty closest galaxies $\left(\rho_{20} / \rho_{\text {ave }}\right)$ and a separation between the target and the closest galaxy $\left(r_{p} / r_{n e i, v i r}\right)$ were adopted for the final set. We used a IRAF/ELLIPSE task to derive the bugle component ellipticity for the final sample. Finally we have 3173 galaxies including 2923 non-starburst, 250 nuclear starburst galaxies. Here we use E that indicates disk ellipticity subtracted from bulge ellipticity in order to consider disk inclination.

\section{Results}

We find a moderate, but clear correlation between the nuclear star formation and non-axisymmetric shape of bulges (Figure 1). Specifically, this relation intensifies in the galaxies with a high bulge fraction $(0.15<\mathrm{B} / \mathrm{T}<=0.41)$ corresponding to the early type compared to the low bulge fraction galaxies. In galaxies with a clear bulge, the relation sustains; however, it weakens in the galaxies with a prominent disk. Also, galaxies in low density environments have a higher fraction of starbursts as ellipticity increases compared to the high density galaxies. This suggests that the relation is maintained in the low density region $\left(\rho_{20} / \rho_{\text {ave }}<5\right)$, but the external factors disintegrate the relation in a group or a cluster environment $\left(\rho_{20} / \rho_{\text {ave }}>5\right)$. Even on the point of view of the small-scale environment, the dependence grows in isolated galaxies $\left(r_{p}>r_{n e i, v i r}\right)$, but it drops in interacting galaxies $\left(r_{p}<r_{n e i, v i r}\right)$. In this case, flying by or merging neighboring galaxies could reduce the dependency. In summary, our results show statistically that nuclear starbursts have a dependency on the elongated bulges with a large number of observation data. This relationship is clear in early type galaxies and those in low density and isolated region. 


\section{References}

Abazajian, K. N. et al. 2009, ApJS 182, 543

Choi, Y.-Y., Han, D.-H., \& Kim, S. S. 2010, JKAS 43, 191

Park, C. \& Choi, Y.-Y. 2009, ApJ 691, 1828

Simard, L., Mendel, J. T., Patton, D. R., Ellison, S. L., \& McConnachie, A. W. 2011, ApJS 196, 11 